\title{
Producción y comercialización de tortillas y su impacto en el ingreso de las familias
}

Production and commercialization of tortillas and its impact on the income of families

AUTORES: $\quad$ Rodríguez Bravo Argenis Gabriel ${ }^{1}$

Albán Mendoza Jenny Cecilia 2

DIRECCIÓN PARA CORRESPONDENCIA: Universidad Estatal del Sur de Manabí, Km 1 ${ }^{1 / 2}$ vía Noboa S/N Campus Los Ángeles - Jipijapa- Ecuador.

Fecha de recepción: 20-03-2019

Fecha de aceptación: 12-04-2019

\section{RESUMEN}

La presente investigación analiza el impacto en el ingreso de las familias de la Asociación de Alimentos de la Comuna Sancán del Cantón Jipijapa, a través de la producción y venta de tortillas. La metodología consistió en aplicar encuesta y entrevista a los miembros de la asociación a fin de recabar información relevante, con una investigación descriptiva a partir de técnicas cualitativas y cuantitativas se obtuvieron datos útiles para el desarrollo de la investigación. El análisis de la situación actual indica que sus miembros tienen escasos conocimientos técnicos sobre el mercado en el que se desenvuelven, análisis financieros, seguridad en el acceso a los canales de distribución y de asociatividad, que no les permite alcanzar el desarrollo sustentable para sus familias y comunidad en general. Los resultados indican que la actividad es desempeñada por grupos familiares de hasta 3 o 4 miembros, lo que representa un $80 \%$ en la economía de la comunidad. Se determinó que una planeación estratégica del negocio aliada a un estudio de mercado, ayudarían a los comuneros a fortalecer las ventas y aumentar los ingresos del negocio, esta herramienta permitirá contribuir al bienestar económico y social de esta estructura organizacional.

Palabras claves: Asociación; ingresos; producción; venta;

\footnotetext{
${ }^{1}$ Ing. Comercio Exterior y Negocios Internacionales. MBA. Docente Universidad Estatal del Sur de Manabí, Facultad de Economía. Carrera Comercio Exterior. Manta. Ecuador. E-mail argenis.rodriguez@unesum.edu.ec

${ }^{2}$ Ing. Comercio Exterior y Negocios Internacionales. Magister en Gestión Empresarial. Administradora de Proservid. Investigadora Independiente. Montecristi. Manabí. Ecuador. E-mail: ceci_alban18@ hotmail.com
} 
Argenis Gabriel Rodríguez Bravo, Jenny Cecilia Albán Mendoza

\section{SUMMARY}

The present investigation analyzes the impact on income of the families of the Food Association of the Comuna Sancán del Cantón Jipijapa, through the production and sale of tortillas. The methodology consisted in applying a survey and interview to the members of the association in order to gather relevant information, with a descriptive investigation based on qualitative and quantitative techniques, useful data were obtained for the development of the research. The analysis of the current situation indicates that its members have little technical knowledge about the market in which they operate, financial analysis, security in access to distribution channels and associativity, which does not allow them to achieve sustainable development for their families. and community in general. The results indicate that the activity is carried out by family groups of up to 3 or 4 members, which represents $80 \%$ in the economy of the community. It was determined that a strategic planning of the business together with a market study, would help the comuneros to strengthen the sales and increase the income of the business, this tool will contribute to the economic and social welfare of this organizational structure.

Keywords: (Association; income; production; sale).

\section{INTRODUCCIÓN}

El escaso acceso a fuentes de empleo en nuestro país cada vez es más apremiante, sobre todo para aquellas personas que por diversas circunstancias carecen de formación académica a nivel superior, provocando restricciones a la hora de postular a un empleo. Por esta y otras razones el emprendimiento en Ecuador ha ido creciendo, de tal manera que para el año 2017 según datos del Global Entrepreneurship Monitor (GEM) (ESPAE- ESPOL, 2018) en Ecuador el 29,6\% de la población comprendida entre 18 y 64 años de edad ha emprendido un negocio o posee uno.

Los latinoamericanos según Kantis (2004) de acuerdo a estudios empíricos sostienen que el obtener ingresos superiores, la realización personal, ser independiente laboral y económicamente, llevar sus conocimientos a la ejecución, el estatus social, la tradición familiar, entre otros aspectos, son los motivos más importantes que los lleva a emprender una actividad o negocio propio. De acuerdo a esto lo que para una familia empieza como estrategia para conseguir ingresos, poco a poco se convierten en conglomerado de familias que unen esfuerzos, ideas, 
recursos económicos y sobre todo deseos de superación y bienestar para sus integrantes. En este sentido es el emprendimiento la actividad que realiza la Asociación de alimentos de la Comuna Sacán del Cantón Jipijapa en la provincia de Manabí, dedicada a la elaboración y venta de tortillas de maíz y yuca; que concuerda con la definición que Robbins \& Coulter (2005) otorga al proceso emprendedor, como aquel que ejecuta esfuerzos organizados individual o colectivamente, en busca de oportunidades, con el ánimo de crear valor para lograr la satisfacción de necesidades con desarrollo e innovación.

El negocio que estos comuneros ejecutan con mano de obra localizada dentro del mismo vínculo familiar lo convierte en empresa familiar, puesto que tienen ciertas particularidades como lo menciona Ginebra (2001) de ser administrada intuitivamente, esto lo hace carecer de un conocimiento profesional y elementos formales. Para De la Rosa, Lozano y Ramírez (2009) en muchas ocasiones es necesario efectuar un control por personas externas que no tengan ningún lazo familiar, estos podrán distinguir el papel y aporte que cada miembro realiza, los problemas dentro del contexto familiar, además de servir de apoyo en la misión y dirección del negocio.

Se plantean varias alternativas de solución para problemas dentro del negocio familiar en el gráfico siguiente:

Gráfico 1. Recomendaciones Básicas para Empresas Familiares.

\begin{tabular}{|c|c|c|c|}
\hline $\begin{array}{l}\text { 1.- Establecer reglas para } \\
\text { los empleados familiares } \\
\text { y definir reglas financieras } \\
\text { y de transparencia }\end{array}$ & $\begin{array}{l}\text { 2.- Recurrir al apoyo } \\
\text { profesional }\end{array}$ & $\begin{array}{l}\text { 3.- Cuidar y establecer } \\
\text { relaciones optimas con los } \\
\text { miembros de la familia } \\
\text { mediante comunicación, } \\
\text { respeto y confianza entre } \\
\text { ¿os mismos }\end{array}$ & $\begin{array}{l}\text { 4.- Identificar a } \\
\text { candidatos internos y } \\
\text { externos a la empresa } \\
\text { para puestos claves }\end{array}$ \\
\hline $\begin{array}{l}\text { 5.- Definir el perfil del } \\
\text { sucesor y sus roles }\end{array}$ & $\begin{array}{l}\text { 6.- Reclutar a los } \\
\text { candidatos según sus } \\
\text { capacidades, valores, } \\
\text { experiencia y } \\
\text { conocimientos }\end{array}$ & $\begin{array}{l}\text { 7.- Tener por escrito, } \\
\text { estandarizar e } \\
\text { institucionalizar todos los } \\
\text { procesos administrativos y } \\
\text { operativos de toda la } \\
\text { ornanización }\end{array}$ & $\begin{array}{l}\text { 8.- Identificar los valores } \\
\text { que han llevado al éxito a } \\
\text { la empresa para } \\
\text { incorporarlos al código } \\
\text { genético de la empresa }\end{array}$ \\
\hline $\begin{array}{l}\text { 9.- La educación } \\
\text { profesional en los hijos } \\
\text { apoyará la armonía en } \\
\text { una sucesión futura }\end{array}$ & $\begin{array}{l}\text { 10.- Definir prioridades y } \\
\text { hacer énfasis entre la } \\
\text { familia y el negocio }\end{array}$ & & \\
\hline
\end{tabular}

Fuente: (Fergusón, 2009) y (Jiménez, 2010)

Cada uno de los socios que integran esta asociación se convierten en emprendedores y generadores de ingresos, mejorando de alguna u otra manera el bienestar de sus familias. Para Mendoza \& Leasaski (2010) el vocablo francés entrepreneur (pionero) da origen a la palabra 
Argenis Gabriel Rodríguez Bravo, Jenny Cecilia Albán Mendoza

emprendedor, que no es más que aquella persona que ejecuta actividades o acciones dificultosas, esto según el diccionario de la Academia de la Lengua Española.

Hoy en día la venta de productos alimenticios se considera una actividad económica de gran relevancia para empresas inmersas en esta rama de la industria, sin embargo, la competencia en crecimiento ha generado un factor de mucho análisis, por ello, las empresas deben apostar hacia la industrialización y profesionalización de la manufactura, que sin lugar a duda permitirán obtener productos nutritivos, de buen nivel alimenticio y de calidad mejorada.

El éxito en emprendimientos acerca de alimentos está en crear proyectos innovadores y atractivos que gusten y permiten una fidelización del cliente. Sin embargo, la Investigación y el Desarrollo son términos desconocidos por la mayoría de los propietarios de negocios, que sumado a la escasez del factor económico los lleva a optar por la improvisación o la continuidad de sus ofertas de productos. La venta de bienes alimenticios ha permitido generar fuentes de trabajo, no solo a los dueños de negocios, sino a terceras personas como trabajadores y proveedores, quienes se encuentran localizados generalmente en el mismo nicho comercial.

En la amplia amalgama de la Investigación y el Desarrollo encontramos a la planeación estratégica como punto neurálgico en la sostenibilidad de un negocio, porque permite hacer una reflexión sobre los elementos internos y externos de la organización para posteriormente diseñar las estrategias y los planes de acción, consiguiendo la consecución de las metas trazadas (Sánchéz, 2003). La planeación permite además elaborar las políticas adecuadas, proporcionar los recursos de manera eficiente, y como toda empresa o negocio permite aprender de los errores traduciéndose en mejores desempeños y aprendizaje constante de las dificultades, desafíos, extenuaciones, fortalezas, etc.

La actual investigación enfocó su estudio en analizar el impacto de la producción y comercialización de tortillas en el ingreso de las familias que pertenecen a la comuna Sancán del Cantón Jipijapa, para obtener información precisa se aplicaron técnicas cuantitativas (encuestas) y cualitativas (entrevistas) obteniendo información de las necesidades y problemas que existen en la asociación y posteriormente analizar e interpretar los datos recolectados en relación a las variables de estudio para el desarrollo de la presente investigación.

18 UNESUM-Ciencias. Publicación cuatrimestral. Vol. 3, Año 2019, No. 1 (abril) 


\section{DESARROLLO}

\section{Materiales y métodos}

La investigación empleada y afín a los objetivos planteados fue de tipo exploratoria porque a través de encuestas y entrevistas a los miembros de la asociación de alimentos de la comuna Sancan, se pudo obtener sus opiniones y argumentos permitiendo identificar factores importantes durante el desarrollo de la investigación; y además fue descriptiva al puntualizar información acerca del proceso de producción y comercialización por parte de los miembros de la asociación, las necesidades y problemas que enfrentan al momento de elaborar y comercializar el producto, pudiendo recopilar, analizar e interpretar sistemáticamente las variables de estudio en base a la realidad de la indagación planteada.

Las técnicas utilizadas dentro de la investigación y que permitieron recolectar datos fueron la entrevista realizada al presidente de la Asociación, así como las respectivas encuestas a sus miembros. Para efectuar la entrevista se utilizó un guion establecido que permitió obtener datos relevantes y la base bibliográfica para las encuestas. Las encuestas se diseñaron con la ayuda de un cuestionario en base a preguntas efectuadas a una muestra seleccionada, con el fin de deducir conclusiones sobre la población.

El procesamiento de datos cualitativos y cuantitativos permitió realizar un análisis de los problemas que existen dentro de la asociación y establecer decisiones que beneficien a las familias que conforman la asociación de alimentos de la comuna Sancan.

Para el estudio estadístico de la investigación se consideró a los miembros de la asociación utilizando técnicas de recolección de datos que permitieron realizar un diagnóstico de la situación actual de los comerciantes y posteriormente optar por alternativas que favorezcan los ingresos de las familias de la comuna Sancan.

La población considerada fue el total de miembros de la "Asociación de alimentos de la comuna Sancan” que está compuesta por 78 miembros, quienes están legamente inscritos; de los cuales y según el cálculo de muestras corresponde efectuar a 35 asociados el formulario con las preguntas 
Argenis Gabriel Rodríguez Bravo, Jenny Cecilia Albán Mendoza

planteadas. La muestra optima o intencional es aquella en la que para elegir sus elementos ha intervenido la opinión o intención de la persona encargada de la selección muestral. Como consecuencia de su lógica subjetividad, se puede afirmar que, por lo general, el proceso de muestreo opinático o intencional proporciona muestras más representativas cuanto menor sea el tamaño de las muestras a obtener. (Dominguez, 2016)

\section{Resultados}

El desarrollo de la investigación permitió obtener un análisis preliminar sobre la problemática planteada como es la influencia de la producción y comercialización de alimentos en el ingreso de las familias que conforman la “Asociación de alimentos de la comuna Sancán”, permitiendo descubrir información previa no identificada.

Los datos recabados a través de entrevista y encuestas se enmarcan en aspectos primordiales dentro del contexto de la producción y comercialización que realiza la asociación en estudio, mencionando que los asociados dentro de sus puntos de ventas ofrecen el producto estrella que son las tortillas de yuca o de maíz, pero que adicionalmente comercializan bienes complementarios como tazas con café, botellas de agua, jugos y gaseosas. La competencia se lleva de manera sana, como asociación busca equilibrio entre el beneficio de cada uno de sus socios contando con estrategias previstas tales como un precio estándar de la tortilla; lo que marca la diferencia en algunas ocasiones es la calidad del producto, es decir al hablar de bien alimenticio el contraste destaca el sabor, la textura, entre otros elementos; puesto que no todos realizan el mismo proceso e ingredientes para la realización del producto final.

Se rescató el aporte o apoyo que han recibido de las autoridades locales y provinciales, las cuales han sido básicamente dos: capacitaciones a los miembros en atención al cliente, la autogestión en la creación de la asociación en el año 2017, para finalmente tener la legalidad correspondiente y de alguna manera como asociados enfrentar cualquier tipo de inconvenientes de manera más sólida y organizada. Hasta el momento no han existido mayores problemas, la debilidad única 
encontrada es el poco tiempo que tienen como asociación, después de esto cada miembro realiza la producción de las tortillas individualmente.

¿Qué tipo de apoyo han recibido por parte del Gobierno para esta iniciativa?

TABLA \# 1

\begin{tabular}{|l|r|r|}
\hline ALTERNATIVAS & FRECUENCIA & PORCENTAJE \\
\hline Recursos Económicos & 0 & $0 \%$ \\
\hline Capacitaciones & 34 & $94 \%$ \\
\hline Otros & 2 & $6 \%$ \\
\hline Ninguno & 0 & $0 \%$ \\
\hline TOTAL & 36 & $100 \%$ \\
\hline
\end{tabular}

FUENTE: Asociación de alimentos de la comuna Sancan del Cantón Jipijapa. ELABORADO: Autores

\section{GRÁFICO \# 2}

¿Qué tipo de apoyo han recibido por parte del Gobierno para esta iniciativa?

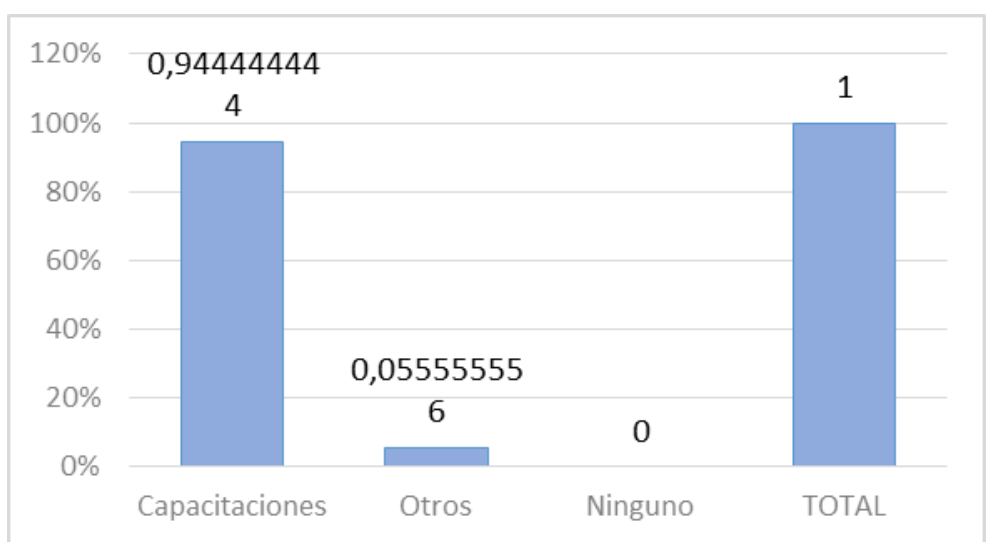

FUENTE: Asociación de alimentos de la comuna Sancan del Cantón Jipijapa.

ELABORADO: Autores

Se trata esencialmente de un negocio familiar que nació hace unas dos décadas atrás, y que hasta la presente ocupa de dos a tres miembros de las familias, lo cual representa un $80 \%$ de ingresos a éstas, considerándolo un aporte positivo puesto que gracias a este trabajo la comunidad tiende a sobresalir. En cuanto al abastecimiento de la materia prima, los proveedores en su mayoría llegan hasta la comunidad para suministrarles el maíz, la yuca y los demás materiales, pero de forma a temporal, considerando además que en diferentes épocas del año ciertos insumos son más escasos que en otras. Como factor positivo se puede destacar que se cuenta con el aprovisionamiento por ejemplo de maíz directamente de agricultores cercanos a la zona. 
Argenis Gabriel Rodríguez Bravo, Jenny Cecilia Albán Mendoza

El cálculo del costo unitario y precio de venta considera incrementos en los valores de insumos principalmente la materia prima, anteriormente vendían las tortillas a precio unitario de $\$ 0.25$, hoy en día el mismo asciende a $\$ 0.35$ centavos por unidad y tres tortillas por un dólar lo que genera un margen de rentabilidad del $50 \%$ por unidad, aproximadamente. Sin embargo, dentro de la organización se presentan problemas que están relacionados con la determinación del costo de los productos, esto se debe a que la materia prima que se utiliza para la elaboración de sus productos tiene tendencia a elevar sus precios volviéndose complicado la determinación del precio del producto terminado.

¿Cuál es el principal problema en la asociación, ya sea a nivel de producción administrativo?

TABLA \# 2

\begin{tabular}{|l|r|r|}
\hline ALTERNATIVAS & FRECUENCIA & PORCENTAJE \\
\hline $\begin{array}{l}\text { Determinación de Costo de } \\
\text { Productos }\end{array}$ & 23 & $64 \%$ \\
\hline Determinación de P.V.P & 11 & $30 \%$ \\
\hline Otros & 2 & $6 \%$ \\
\hline TOTAL & $\mathbf{3 6}$ & $\mathbf{1 0 0 \%}$ \\
\hline
\end{tabular}

FUENTE: Asociación de alimentos de la comuna Sancan del Cantón Jipijapa.

ELABORADO: Autores

GRÁFICO \# 3

¿Cuál es el principal problema en la asociación, ya sea a nivel de producción administrativo?

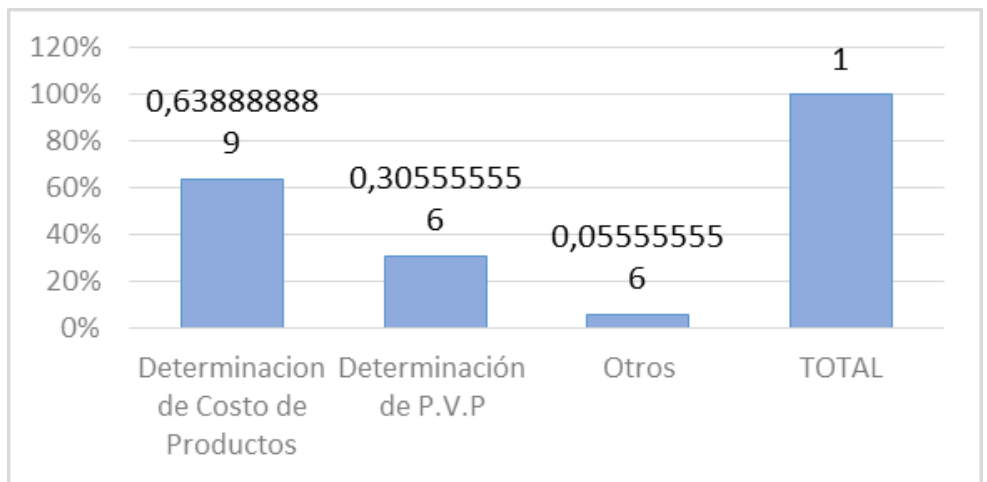

FUENTE: Asociación de alimentos de la comuna Sancan del Cantón Jipijapa.

ELABORADO: Autores

22 UNESUM-Ciencias. Publicación cuatrimestral. Vol. 3, Año 2019, No. 1 (abril) 
La comercialización de las tortillas se efectúa de manera directa con el cliente, los puntos de ventas se localizan a lo largo de la carretera principal que conecta a la provincia de Manabí con Guayas; este punto estratégico les otorga mayor afluencia de personas que transitan diariamente, además de la accesibilidad y contacto con diversas personas a cualquier hora durante el día. Aunque también se realiza de manera ambulatoria (recorriendo los buses que circulan por la vía), ante lo cual los miembros de la asociación consideran que la dirigencia debe canalizar propuestas para su seguridad ya que están expuestos a la delincuencia común.

\section{Discusión}

Para los integrantes de la Asociación de alimentos de la comuna Sancán del Cantón Jipijapa, es muy importante el trabajo que efectúan día a día puesto que representa el sustento de sus familias, al ocupar gran parte de mano de obra se benefician aún más porque al tiempo de permitirles generar ingresos económicos también les asegura estar pendiente de sus hogares sin tener que movilizarse largas distancias para laborar. El valor comercial de la comunidad lo genera el lugar estratégico de ubicación, donde las personas pueden consumir un alimento fresco hecho a base de productos de la zona y su degustación puede ir complementada con una taza de café caliente, preparada en el mismo lugar, a un precio muy económico; a diferencia del consumo de productos industrializados, con escaso valor nutritivo y a precios altos que ofrecen las cadenas comerciales. Por lo tanto, se puede decir que el precio, la calidad del producto y el servicio son aspectos muy significativos.

Es importante mencionar que los comuneros también reciben ingresos adicionales por las ventas de productos complementarios a las tortillas y cuando algún cliente requiere cantidades mayores también se les despacha, lo que genera nuevos ingresos ocasionales. Pese a todos los beneficios expuestos, dentro de la asociación existen problemas vinculados a aspectos financieros y que son requeridos para la compra de nuevos equipos de trabajo como hornos industriales, sillas, mesas, entre otros utensilios; se requiere que la asociación busque mecanismos a fin de lograr estos beneficios. Otro de los problemas es la escasa producción de materia prima cosechada por los mismos socios y que conlleva a proveerse de otras fuentes con precios más elevados y en ciertas ocasiones la calidad no es la misma. 
Argenis Gabriel Rodríguez Bravo, Jenny Cecilia Albán Mendoza

Como ente organizado desde el año 2017 la asociación no ha logrado consolidarse de tal manera que apliquen otras alternativas y estrategias de producción y venta capaces de llegar a obtener mayores ingresos y fuentes de trabajo. Como se pudo notar la falta de conocimiento por poner un ejemplo en el cálculo de costo de producción y fijación de precio para la venta son aspectos trascendentales en la obtención de mejor rentabilidad y sostenibilidad del negocio. Como sujeto generador principal de trabajo dentro de la comunidad de Sancán, los socios deben aprovechar la vida jurídica y la organización que tienen como asociación para despuntar hacia mejores logros y beneficios para sus integrantes y familias.

El apoyo recibido hasta la presente por organismos e instituciones les ha permitido obtener conocimientos básicos y su legalidad, pero faltaría formarlos aún más en aspectos sencillos, pero al mismo tiempo innovadores y de acuerdo a las necesidades específicas de los mismos, como finanzas básicas, planificación estratégica, emprendimiento, entre otros. Además, es importante inducirlos a la autogestión que como asociación les procura un sinnúmero de programas, alternativas y proyectos que con la asesoría de instituciones sobre todo públicas se pueden conseguir, es cuestión simplemente de unir esfuerzos y aptitudes positivas.

Desarrollar un efectivo análisis de las cinco fuerzas que presenta Michel Porter permite enfocar puntos críticos y oportunidades dentro de una industria, tal como lo observamos en el gráfico 4.

\section{GRÁFICO \# 4}

\section{Las cinco fuerzas que moldean la competencia en un sector}

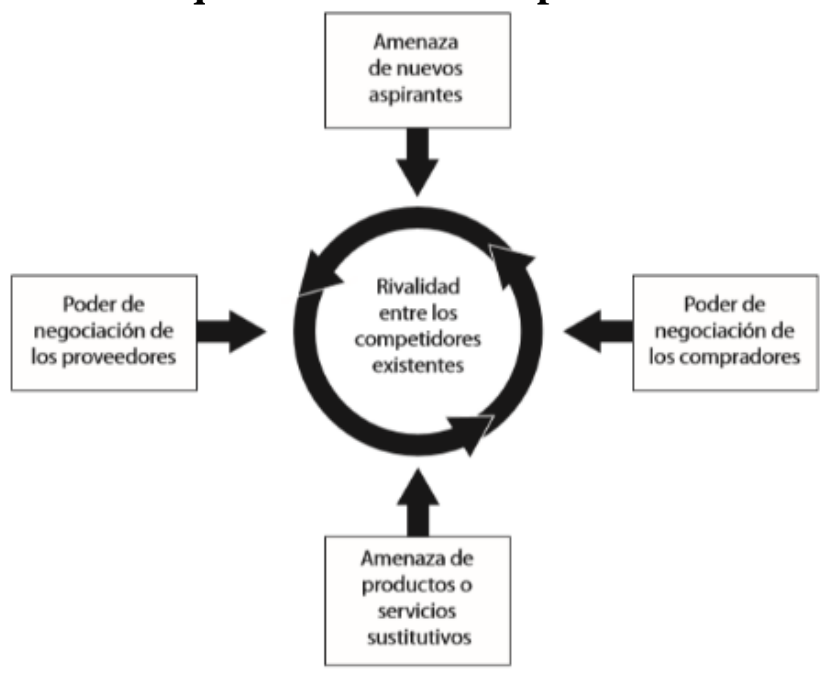

FUENTE: (Porter, 2017, pág. 32). 
El objetivo que persigue este tipo de análisis es percibir los componentes dentro de la organización tanto de competencia como de rentabilidad; los puntos favorables dentro del análisis repercuten en la fijación de precios, costos e inversión óptima; por ejemplo la primera fuerza que ejerce mucha presión es la entrada de nuevos competidores al mercado, esta situación genera efectos, para considerar que el ingreso a la industria analizada es fácil o difícil habría que determinar montos de la inversión requerida, período de recuperación de la inversión, facilidad de mano de obra e insumos, competidores actuales, etc. Entre que las barreras de entrada sean más fáciles la rentabilidad de la industria en términos generales se vuelve vulnerable (Porter, 2017).

Para Hernández (2010) la cantidad de proveedores disponibles, el volumen de compras, la variedad de materia prima sustituta determina cuánto poder se tenga para conseguir precios mínimos, entregas inmediatas de insumos, calidad de los mismos, flexibilidad en los pagos, etc. Así mismo de importante es el poder de negociación de los clientes, en esta fuerza influyen la cantidad adquirida, la concentración de clientes, la diferenciación o el plus que tenga el producto, el conocimiento de productos sustitutos por parte del cliente, etc.

La rentabilidad de una industria también se ve afectada por la existencia de productos sustitutos (Baena, Sánchez, \& Montoya, 2003), en este sentido habría que considerar precios relativos, facilidad al acceso de los mismos, rendimiento y calidad del sustituto y los costos que implica el cambio del producto. Y el punto central de este análisis es la rivalidad entre los competidores existentes, si la industria en análisis compite fuertemente en precios, en publicidad, en calidad, en innovación, es decir, cuando la competencia es más fuerte se considera a la industria menos rentable y por ende con menos posibilidad de ingreso de nuevos competidores.

\section{Gráfico 5. Análisis de las fuerzas de Porter en la Asociación de alimentos de la comuna} Sancán. 


\begin{tabular}{|c|c|c|c|}
\hline \multirow{4}{*}{$\begin{array}{l}\text { Poder de los } \\
\text { compradores }\end{array}$} & \multirow{7}{*}{$\begin{array}{l}\text { Los compradores tienen más poder } \\
\text { cuando: } \\
\text { Los vendedores son pocos y pequeños y los } \\
\text { compradores pocos y grandes. } \\
\text { Los compradores adquieren grandes } \\
\text { cantidades. } \\
\text { Un comprador individual es un gran cliente. } \\
\text { Los compradores pueden cambiar } \\
\text { proveedores a bajo costo. } \\
\text { Los compradores compran de múltiples } \\
\text { vendedores a la vez. } \\
\text { Los compradores pueden integrarse } \\
\text { fácilmente hacia atrás. } \\
\\
\text { Los competidores entrantes (a la } \\
\text { industria) amenazan a las compañías } \\
\text { establecidas. } \\
\text { Barreras al ingreso: } \\
\text { Lealtad de marca } \\
\text { Ventajas absolutas de costo } \\
\text { Economías de escala } \\
\text { Costos ínter cambiantes } \\
\text { Normativas Gubernamentales } \\
\text { Las barreras al ingreso reducen la amenaza } \\
\text { de nueva competencia }\end{array}$} & & $\mathbf{x}$ \\
\hline & & & $\mathbf{x}$ \\
\hline & & $\mathbf{x}$ & \\
\hline & & & $\mathbf{x}$ \\
\hline \multirow{3}{*}{$\begin{array}{l}\text { Nuevos competidores / } \\
\text { potenciales }\end{array}$} & & $\mathbf{x}$ & \\
\hline & & & $\mathbf{x}$ \\
\hline & & $\mathbf{x}$ & \\
\hline \multirow[t]{2}{*}{$\begin{array}{l}\text { Rivalidad con } \\
\text { establecidos }\end{array}$} & $\begin{array}{l}\text { La intensidad de la rivalidad competitiva } \\
\text { en una industria surge de: } \\
\text { La estructura competitiva de la industria. } \\
\text { Las condiciones de la demanda (crecimiento } \\
\text { o declinación) en la industria. }\end{array}$ & $\mathbf{x}$ & \\
\hline & $\begin{array}{l}\text { El tamaño de las barreras de salida en la } \\
\text { industria. }\end{array}$ & & $\mathbf{x}$ \\
\hline \multirow{3}{*}{$\begin{array}{l}\text { Poder de los } \\
\text { proveedores }\end{array}$} & $\begin{array}{l}\text { Los proveedores tienen poder de } \\
\text { negociación si: } \\
\text { Sus productos tienen pocos sustitutos y son } \\
\text { importantes para los compradores. } \\
\text { La industria del comprador no es un cliente } \\
\text { importante para el proveedor. }\end{array}$ & $\mathbf{x}$ & \\
\hline & $\begin{array}{l}\text { La diferenciación hace costoso que los } \\
\text { compradores cambien de proveedor. }\end{array}$ & $\mathbf{x}$ & \\
\hline & $\begin{array}{l}\text { Los proveedores pueden integrarse hacia } \\
\text { delante y competir con los compradores, y } \\
\text { estos no pueden integrarse hacia atrás para } \\
\text { llenar sus necesidades. }\end{array}$ & $\mathbf{x}$ & \\
\hline Productos sustitutos & La amenaza competitiva de los productos & $\mathbf{x}$ & \\
\hline
\end{tabular}




\begin{tabular}{|l||l||l||}
\hline & $\begin{array}{l}\text { sustitutos incrementa conforme se acercan en } \\
\text { su capacidad de llenar necesidades de los } \\
\text { clientes. }\end{array}$
\end{tabular} \mid

Fuente: Elaborado por autores.

\section{CONCLUSIONES}

La producción y comercialización de tortillas elaboradas por los miembros de la Asociación de Alimentos de la Comuna Sancán del cantón Jipijapa, que actualmente cuenta con 78 miembros activos, genera muchas plazas de trabajo lo que a su vez aporta a los ingresos familiares de los habitantes, por lo que es fundamental el aporte constante de entidades públicas, locales y nacionales. Se identificaron ciertas necesidades técnicas y financieras para implementar mejoras en sus unidades de negocios. La seguridad en las ventas ambulatorias es otra de las necesidades identificadas.

Al analizar el desempeño realizado se puede reflejar que su impacto económico es positivo, por las mismas razones que genera plazas de trabajo dentro de la comuna, y que en cada punto de venta de las tortillas intervienen tres o más miembros de la familia, el cual representa un $80 \%$ en la economía de la comunidad. La búsqueda de autogestión y apoyo institucional son prioridades y elementos que generan mejores resultados y se mantienen en el tiempo, es ideal conseguir la asesoría del Instituto de Economía Popular y Solidaria, Superintendencia de Control de Poder de Mercado, Pro Ecuador, y diversas organizaciones que ofrecen colaboración gratuita y variadas alternativas para colocar el producto con mejores precios y cantidades vendidas.

Al contar con estructura jurídica, esta asociación podría aplicar mecanismos como el análisis de las cinco fuerzas de Porter, amenaza de nuevos competidores, competidores existentes (fuera de la asociación), productos sustitutos, poder de negociación con los clientes y poder de negociación con los proveedores (Baena, Sánchez, \& Montoya, 2003).

Con una planificación integral y estratégica, con mayores conocimientos, mejores oportunidades productivas y comerciales y la unión de todos sus miembros, la Asociación de Alimentos de la 
Argenis Gabriel Rodríguez Bravo, Jenny Cecilia Albán Mendoza

Comuna Sancán del cantón Jipijapa logrará ser un referente de emprendimiento familiar y dinamizador de la economía del sector y de la provincia en general.

\section{REFERENCIAS BIBLIOGRÁFICAS}

Baena, E., Sánchez, J., \& Montoya, O. (2003). EL ENTORNO EMPRESARIAL Y LA TEORÍA DE LAS CINCO FUERZAS COMPETITIVAS . Scientia et Technica , 61-66.

De la Rosa, A., Lozano, O., \& Ramirez, J. (2009). Organización, empresa y familia: de la empresa familiar a la organización familiar. Gestión y Estrategia, 17-36.

Dominguez, I. (14 de noviembre de 2016). ENCICLOPEDIA FINANCIERA. Recuperado el 20 de ABRIL de 2019, de ENCICLOPEDIA FINANCIERA: https://www.enciclopediafinanciera.com/diccionario/muestra-opinatica.html

ESPAE- ESPOL. (2018). Global Entrepreneurship Monitor Ecuador 2017. Guayaquil: ESPAE GRADUATE SCHOOL OF MANAGEMENT .

Fergusón, I. (19 de MAYO de 2009). CNN Expansión. Recuperado el 22 de ABRIL de 2019, de https://expansion.mx/emprendedores/2009/05/18/como-lograr-el-exito-de-tu-empresa

Ginebra, J. (2001). Las empresas familiares: su dirección y su continuidad. México: Panorama.

Hernández, J. (S/D de S/M de 2010). EL MODELO DE COMPETITIVIDAD DE LAS CINCO FUERZAS DE PORTER. Recuperado el 18 de ABRIL de 2019, de http://monografias.umcc.cu/monos/2010/INDECO/mo1068.pdf

Jiménez, A. (8 de ABRIL de 2010). CNN EXPANSIÓN. Recuperado el 22 de ABRIL de 2019, de https:/expansion.mx/mi-carrera/2010/04/07/como-elegir-al-sucesor-de-mi-empresa

Kantis, H. (2004). Desarrollo emprendedor: América Latina y la experiencia Internacional. Bogotá: Nomos.

Mendoza, J., \& Leasaski, D. (2010). Determinantes del proceso de emprendimiento empresarial femenino en el Perú. Pensamiento Crítico, 57-70.

Porter, M. (2017). Ser competitivo . Barcelona: Ediciones Deusto.

Robbins, S., \& Coulter, M. (2005). Administración. México D.F.: Prentice Hall.

Sánchéz, J. (2003). ESTRATEGIA INTEGRAL PARA PYMES INNOVADORAS. REVISTA ESCUELA DE ADMINISTRACIÓN DE NEGOCIO, 34-45. 